\title{
Serological and genotyping study of rare cis-AB01/001 in the area of Inner Mongolia - with a case report
}

\author{
Chunhai Wang ${ }^{1}$, Wenmei Wang ${ }^{* 2}$, Hualiang Huang ${ }^{1}$ \\ ${ }^{1}$ Department of Blood Transfusion, Baogang Hospital, Baotou, Inner Mongolia, China \\ ${ }^{2}$ Department of Critical Care Medicine, the First Affiliated Hospital of Baotou Medical College Inner Mongolia University of \\ Science and Technology, Baotou, Inner Mongolia, China
}

Received: November 30, 2019

Accepted: February 25, $2020 \quad$ Online Published: April 19, 2020

DOI: $10.5430 /$ dcc.v6n $3 p 4$

URL: https://doi.org/10.5430/dcc.v6n3p4

\begin{abstract}
The antigen gene of $\mathrm{ABO}$ blood group system, called $\mathrm{ABO}$, is located on human chromosome 9, with a total length of $19.5 \mathrm{~kb}$. It is the first blood group system found by human beings. ${ }^{[1]} \mathrm{ABO}$ blood group subtypes are formed by $\mathrm{ABO}$ genovariation, i.e., gene $A$ variation for $A$ subtype, gene $B$ variation for $B$ subtype and gene $O$ variation for new $O$ alleles. ABO subtypes contain $A 3$, Ax, Ael, Aw, Am, B3, Bx, Bel, Bw, cis-AB, B (A). Generally, an individual with AB blood group has an A allele on one chromosome, with $\mathrm{B}$ allele on its paired chromosome. This phenomenon is called trans-AB. However, cis- $\mathrm{AB}$ is a unique ABO phenotype that $\mathrm{A}$ and $\mathrm{B}$ alleles are located on the same chromosome, so that it can be inherited by the next generation. ${ }^{[2]}$ This special mode of inheritance often causes a discrepancy of ABO blood grouping and then reduces the effectiveness and safety of blood transfusion. Therefore, to accurately identify the blood group of cis-AB is a precondition for the safety of blood transfusion. ${ }^{[3]}$ The serological and genotyping analysis on a case of cis- $\mathrm{AB}$ patient in our hospital is reported as follows.
\end{abstract}

Key Words: cis-AB subtype, Genotyping, Blood-group serology, Gene sequencing

\section{DATA AND METHODS}

\subsection{General information}

A male patient, 61 years old, with right innominate bone pain and 2-year limitation of motion, was proposed to be given a scheduled operation, with a past history of blood transfusion. Sufficient blood was prepared for blood transfusion as normal before operation. Ortho AutoVue automatic blood grouping and matching analyzer system showed the following identification results: direct grouping indicated AB subtype, but indirect grouping indicated A subtype instead, with a discrepancy of blood grouping. The further serological examination for blood grouping was made by recollecting the blood sample from the patient and sending it to Nobel Prize R\&D Institute of Jiangsu ZOJIWAT Biotech Co., Ltd. for ABO genotyping and gene sequencing (Sample No.: W126).

\subsection{Instruments and reagents}

Ortho AutoVuet automatic blood grouping and matching analyzer, Taiwan BASO centrifuge, water bath; Anti-A, Anti-B and $\mathrm{RhD}(\mathrm{IgM})$ blood grouping reagents, screened red blood cells, Anti-H reagents and Anti-A1 reagents (SHPBC); ABO indirect grouping reagents (Changchun Brother Biotech Co., Ltd.); Anti-A and Anti-B reagents (France); Human red blood cell $\mathrm{ABO}$ genotyping kits (fluorescence PCR, Batch No.: 20180226, Jiangsu ZOJIWAT Biotech Co., Ltd.), and all reagents above were used within the available period.

\footnotetext{
*Correspondence: Wenmei Wang; Email: yfyicuwm@126.com; Address: Department of Critical Care Medicine, the First Affiliated Hospital of Baotou Medical College Inner Mongolia University of Science and Technology, Baotou, Inner Mongolia, China.
} 


\subsection{Methods}

\subsubsection{ABO blood group serological examinations}

Serological examinations such as ABO blood grouping, Anti$\mathrm{H}$ determination, Anti-AB \& antibody screening and saliva test were performed strictly in accordance with relevant literatures. ${ }^{[4,5]}$

\subsubsection{ABO genotyping and gene sequencing}

Nobel Prize R\&D Institute of Jiangsu ZOJIWAT Biotech Co., Ltd. was engaged in the ABO blood grouping.

\section{RESULTS}

\subsection{The results of $\mathrm{ABO}$ blood grouping}

The results showed that direct grouping indicated AB genotype (B antigens showed a low expression intensity in a mixed field) and indirect grouping indicated A genotype, with a discrepancy of blood grouping. It was preliminarily determined that $\mathrm{AB}$ subtype generated anti-B or attenuated $\mathrm{B}$ antigens with alloantibodies. In addition, in direct grouping, 0 reaction to anti-A1, 4+ reaction to anti- $\mathrm{AB}, 3+$ reaction to anti-H; in indirect grouping, $1+$ reaction to $B$ cells. It was indicated that this type of antibody was probably anti-B antibody. In combination with the medical history, the patient was suspected to be cis- $\mathrm{AB}$, which was required to be confirmed by performing a gene test. The results were shown in Table 1.

\subsection{Antibody screening test}

Both saline tube method and anti-human globulin test showed negative results; and indirect grouping showed $1+$ reaction to $\mathrm{B}$ cells from 3 donors and negative to $\mathrm{O}$ cells from 3 donors respectively. Combined with these two tests, it can be determined that the antibody in this case of patient is a type of irregular anti-B instead of non-ABO alloantibody.

Table 1. The results of serological examination for ABO blood grouping

\begin{tabular}{|c|c|c|c|c|c|c|c|c|c|c|c|c|}
\hline \multirow{2}{*}{$\begin{array}{l}\text { Blood Group } \\
\text { Reagent }\end{array}$} & \multirow[b]{2}{*}{ Anti-A } & \multirow[b]{2}{*}{ Anti-B } & \multirow[b]{2}{*}{ Anti-A1 } & \multirow[b]{2}{*}{ Anti-AB } & \multirow[b]{2}{*}{ Anti-D } & \multirow{2}{*}{$\begin{array}{l}\text { A1 } \\
\text { cells }\end{array}$} & \multirow{2}{*}{$\begin{array}{l}\text { B } \\
\text { cells }\end{array}$} & \multirow{2}{*}{$\begin{array}{l}\text { O } \\
\text { cells }\end{array}$} & \multirow{2}{*}{$\begin{array}{l}\text { Self } \\
\text { Control }\end{array}$} & \multicolumn{3}{|c|}{ Anti-H } \\
\hline & & & & & & & & & & Patient & $\begin{array}{l}\text { Adult B Cell } \\
\text { Control }\end{array}$ & $\begin{array}{l}\text { Adult O Cell } \\
\text { Control }\end{array}$ \\
\hline $\begin{array}{l}\text { Micro-column } \\
\text { glass bead } \\
\text { reagent card }\end{array}$ & $4+$ & $\mathrm{mf}$ & / & I & $4+$ & 0 & $1+$ & / & / & I & / & I \\
\hline $\begin{array}{l}\text { Saline tube } \\
\text { method }\end{array}$ & $4+$ & $1+$ & 0 & $4+$ & $4+$ & 0 & $1+$ & 0 & 0 & $3+$ & $1+$ & $4+$ \\
\hline
\end{tabular}

Notes. $\mathrm{mf}$ stands for mixed field, 0 for no reaction

\subsection{The results of saliva test}

The results showed as secretory type, and the saliva sample contained A substance, $\mathrm{H}$ substance and weakly secretory B substance.

\subsection{The results of $\mathrm{ABO}$ genotyping}

The genotyping of the sample (W126) by fluorescence PCR displayed the following results: Type $\mathrm{A}$ indicated positive bands, which was identified as A rather than A205; Type OT and Type $\mathrm{O} 01$ indicated positive bands, which was identified as $\mathrm{O} 01$ (see Figure 1). The result of ABO genotyping: $\mathrm{A} / \mathrm{O} 01$.

\subsection{The results of $\mathrm{ABO}$ gene sequencing}

The results of gene sequencing for this sample (W126) showed no mutation at 297A/A of exon 6 and G deletion at 261 , which resulted in the appearance of consecutive interference peaks. The two points mentioned were considered to be the specific sequences of $\mathrm{O} 01$ subtype. Nevertheless, this sample also showed two mutations at $467 \mathrm{C}>\mathrm{T}$ and $803 \mathrm{G}>\mathrm{C}$ of exon 7, which conformed to the genetic characteristics of cis-AB01 genotype. According to the sequencing results, the genotype of this sample was speculated to be cis-AB01/O01.
See Figure 2 and Figure 3 for the complete sequencing results of $\mathrm{ABO}$ genotyping.

\subsection{Final determinative results}

On the basis of serological and genetic examinations, it can be determined that the blood group in this case should be cis- $\mathrm{AB}$, whose genotype conforms to cis-AB01/O01.

\section{Discussion}

cis-AB is an extremely rare subtype, which has a very low probability in the population (about 1/580,000 1/170,000). Some literatures show that, in general, there are about 14 cases of cis-AB subtype in 1,000,000 Japanese cases, with a ratio of $0.0014 \%$, while in 112,710 examined cases of $\mathrm{AB}$ genotype, the ratio is about $0.012 \% .{ }^{[6]}$ In 1965 , Yamaguchi et al. ${ }^{[7]}$ reported that, in A2B3 subtype, both A2 allele and B3 allele were considered to be located on the same chromosome. When A or B glycosyltransferase gene haplotype allele shows one-point or multi-point mutation, it will cause glycosyltransferase with this gene coding to simultaneously catalyze two types of substrates added to L-Fucose to produce $\mathrm{A}$ and $\mathrm{B}$ antigens. The resulting $\mathrm{AB}$ subtype is called cis-AB. ${ }^{[8]}$ There are two possibilities for the appearance of 
cis- $\mathrm{AB}$ : one is unequal genetic crossing over, in which A A and B antigens. Since ABO genotypes were cloned and allele on one chromosome combines with $\mathrm{B}$ allele on the paired chromosome to form a novel cis-AB subtype with normal genetic information; the other is the mutation at A allele or $\mathrm{B}$ allele, in which the gene coding products contain both sequenced in 1990, the molecular genetic model of cis-AB subtype has been gradually studied and revealed by some scholars. The main cause for cis- $\mathrm{AB}$ phenotype is related to the point mutation of A or B allele on exon 7. ${ }^{[2]}$
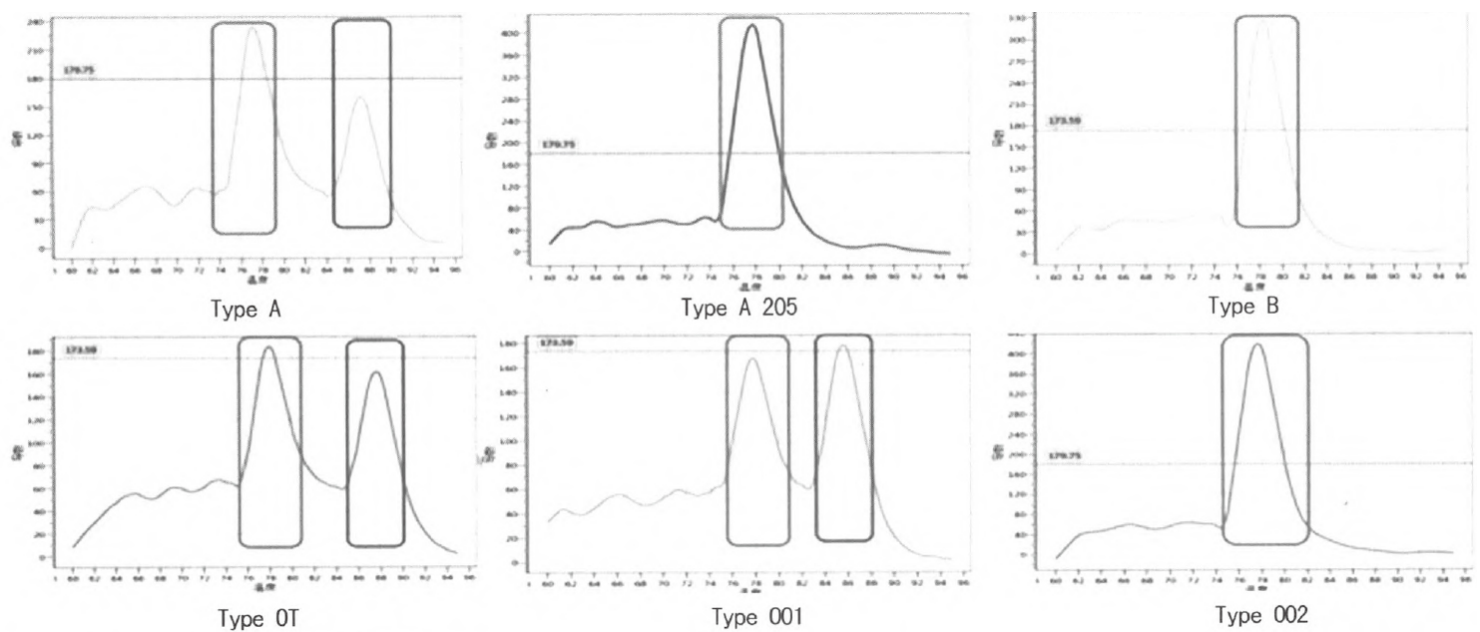

Figure 1. Genotyping results

Blue frame line stands for internal reference, and red frame line stands for positive band

\begin{tabular}{|c|c|c|c|c|c|c|c|c|c|c|c|c|c|}
\hline \multirow{2}{*}{ Reference } & \multicolumn{2}{|c|}{ Exors 6} & \multirow[b]{2}{*}{467} & \multirow[b]{2}{*}{526} & \multirow[b]{2}{*}{$6-46$} & \multirow[b]{2}{*}{657} & \multicolumn{3}{|c|}{ Exan 7} & \multirow[b]{2}{*}{796} & \multirow[b]{2}{*}{803} & \multirow[b]{2}{*}{829} & \multirow[b]{2}{*}{930} \\
\hline & 261 & 297 & & & & & 681 & 703 & 771 & & & & \\
\hline A1. 01.1 & $\mathbf{G}$ & $A$ & G & $\mathrm{c}$ & $\mathrm{T}$ & $\mathrm{C}$ & G & 6 & c & c & 6 & a & $\mathbf{a}$ \\
\hline A102 & a & A & $\mathbf{T}$ & c & $T$ & c & $\mathbf{a}$ & G & c & c & G & a & $\mathbf{G}$ \\
\hline B101 & a & a & c & G & $\mathrm{T}$ & T & 8 & A & C & A & c & a & A \\
\hline 001 & deol & A & $\mathrm{c}$ & c & T & c & $\mathbf{G}$ & G & c & c & G & a & $a$ \\
\hline 002 & $d=1$ & a & c & c & A & c & A & G & 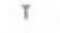 & c & G & A & 6 \\
\hline $\mathrm{Cls}-\mathrm{ABO1}$ & a & A & $\mathrm{I}$ & C & $\mathrm{T}$ & c & $\mathbf{a}$ & $\mathbf{G}$ & c & C & c & G & $G$ \\
\hline W126 & $A B$ & AA & GT & GC & TT & $c 0$ & es & GG & $\mathrm{cc}$ & CC & $\mathrm{cos}$ & 00 & 60 \\
\hline$W 126$ - Cis - $A B 01$ & G & A & $T$ & C & $T$ & c & $a$ & 0 & c & c & C & $a$ & $\mathbf{B}$ \\
\hline W126-001 & $d e 1$ & A & C & c & $\boldsymbol{T}$ & c & $G$ & 6 & c & c & $G$ & $\mathbf{a}$ & G \\
\hline
\end{tabular}

Figure 2. The complete sequencing results of $\mathrm{ABO}$ genotyping

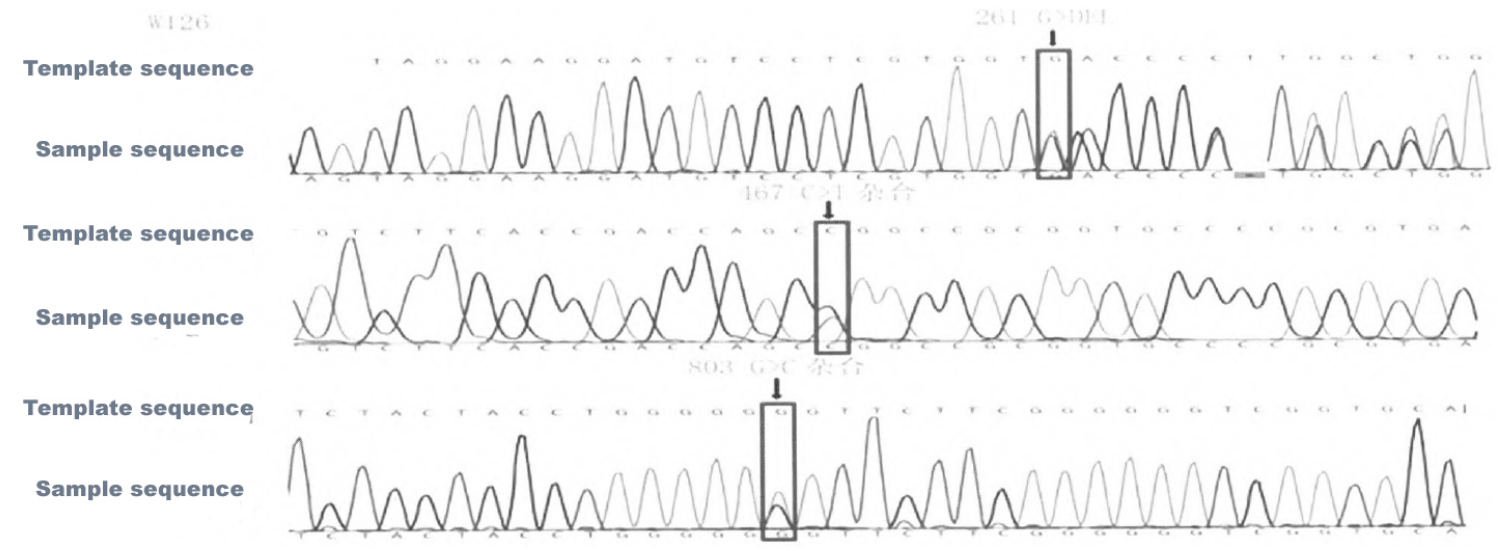

Figure 3. Mutation Point Screenshot 
So far, 8 subtypes of alleles for cis-AB genotype have been found, they are: 01, 02, 03, 04, 05, 06, 07, 08 and 01var. ${ }^{[9]}$ The main characteristics of cis-AB01 are point mutations at $803 \mathrm{G}>\mathrm{C}$ and $467 \mathrm{C}>\mathrm{T} .{ }^{[10]}$ This case of patient shows no mutation at 297A/A of exon 6 and $\mathrm{G}$ deletion at 261, which leads to the appearance of consecutive interference peaks before 261. The two points mentioned were considered to be the specific sequences of O01 subtype. Nevertheless, this patient also shows two mutations at $467 \mathrm{C}>\mathrm{T}$ and $803 \mathrm{G}>\mathrm{C}$ on exon 7, which conform to the genetic characteristics of cis-AB01 genotype. The exon sequence conforms to the heterozygous characteristics of cis-AB01/O01, i.e., cis-AB01 and $\mathrm{O} 01$ constitute a heterozygote, whose genotype and sequencing result should be cis-AB01/O01. Cis-AB01/O01 subtype accounts for $66.7 \%$ of the total cis-AB genotypes. ${ }^{[1]}$ In cis- $A B / O$ genotype, one is haplotype $A B$ allele, the other is haplotype $\mathrm{O}$ allele. The serological results of cis- $\mathrm{AB}$ blood group have certain characteristics. When cis- $\mathrm{AB}$ and $\mathrm{O}$ alleles constitute a heterozygote, the serological results generally show the genotype as A2B3: (1) even though A antigen on cis- $A B$ cell is often considered as $A 2$, but the intensity of $A$ antigen is higher than that of $\mathrm{A} 2 \mathrm{~B}$ and lower than that of A1B; (2) almost all of cis-AB cells weakly express B antigenicity unexceptionally, which is similar to B3 phenotype; have a strong reaction to immunological A type serum, and weakly agglutinate or not agglutinate with serum anti-B; (3) cis- $\mathrm{AB}$ cells have a high $\mathrm{H}$ antigenicity and a moderate reaction (reach up to $3+$ ) to O cells; (4) weak anti-B always exists in cis-AB human blood serum. ${ }^{[5]}$ In the serological examination for blood grouping: in direct grouping, the results show the agglutination of anti-A (4+) and no agglutination of anti-A1 (0), which indicates the existence of A2 antigens, with weak reaction and a mixed field $(\mathrm{mf})$ reaction to anti-B; in indirect grouping, the results show weak reaction to anti-B $(+)$. In the light of antibody screening and the results of serological examinations for $\mathrm{B}$ cells from 3 donors and $\mathrm{O}$ cells from 3 donors in indirect grouping, it can be determined that the antibody is a type of irregular anti-B instead of non-ABO alloantibody, and the intensity of anti-H is obviously higher than that in normal B type, which indicates the existence of B3 antigens. The sample from this case of patient shares the same serological characteristics as A2B3. ${ }^{[5]}$

It is important to differentiate A2B3 from the following two situations in the serological characteristics of cis-AB: (1) the difference from $\mathrm{ABx}$ or $\mathrm{AxB}:{ }^{[11]}$ because cis- $\mathrm{AB}$ allele and other normal A or B allele constitute a heterozygote, $\mathrm{ABx}$ or $\mathrm{AxB}$ often has an effect of antigen dose, with no enhancement of anti-H intensity or weak anti-H intensity, the agglutination intensity of anti- $\mathrm{H}$ is generally lower than that in the reaction of $\mathrm{O}$ cells to anti- $\mathrm{H}^{\left[{ }^{[12,13]}\right.}$ (2) the difference from acquired $\mathrm{B}$ : acquired $\mathrm{B}$ is associated with diseases and is only expressed in A type red blood cells, so that the reaction pattern appears to be similar to that in $A B$ type during the process of blood grouping. The individual with acquired B antigens only have anti-B antibodies in the blood, but these antibodies have no reaction to acquired B cells. Besides, acquired B antigens could weaken the antigenicity of $\mathrm{A}$ antigens. Therefore, the antigenicity of A antigens in red blood cells will be enhanced after acquired B antigens disappear. ${ }^{[5]}$

Due to the effects of various factors, it is difficult to identify cis-AB blood group on the ground of serological manifestations, as the existence of weak anti-B antigens may lead to a discrepancy of $\mathrm{ABO}$ blood grouping and then easily result in the erroneous judgment of blood group. During the process of clinical tests, it often appears as the difficulty in blood grouping and cross-matching. If blood transfusion incompatibility occurs, it will cause hemolytic transfusion reactions. As to this case of patient, there are anti-B antibodies in the serum, so that B type red blood cells could not be used in the blood transfusion. It is strongly recommended to transfuse O type washed red blood cells and AB type blood plasma or perform autologous blood transfusion. Considering the indication for blood transfusion in this case completely met the requirements of preoperative autologous blood donation, $400 \mathrm{ml}$ of autoblood was collected from the patient 3 days before operation. Autologous blood transfusion was performed during the surgery, with no postoperative adverse reactions.

According to incomplete statistics, it was the first time to report a case of patient with cis-AB01/O01 subtype in the area of Inner Mongolia. Since this patient didn't consent to the investigation of his genetic conditions, it was unable to make a pedigree study.

In conclusion, it is unable to accurately identify cis-AB subtype according to the serological reaction pattern of cis-AB. It is necessary to make a differentiation by use of molecular biological techniques (such as ABO genotyping). Pedigree study is recommended if applicable. Only to accurately identify cis-AB blood group can guarantee the safety of blood transfusion and prevent from the occurrence of adverse reactions for blood transfusion.

\section{CONFLicts OF INTEREST Disclosure}

The authors declare they have no conflicts of interest. 


\section{REFERENCES}

[1] Hui Z, Yue J, Fang Y, et al. Study on serological and genetic characteristics of inherited Cis AB blood group: a case report of CisAB family. Chinese Journal of Blood Transfusion. 2015; 28(1): 18-21.

[2] Rong G, Zhiyi Z, Yongjun W. Compatibility test in blood transfusion and analysis on intractable cases. Beijing: People's Medical Publishing House; 2018. 20 p.

[3] Sha J, Xiaohong C, Xi L, et al. Study on cisAB and B (A) subgroups in Shanghai blood donors, China. Chinese Journal of Blood Transfusion. 2013; 26(12): 1198-1201.

[4] Yingfu Y, Yusan W, Ziyu S. National Guide to Clinical Laboratory Procedures. Third Edition. Nanjing: Southeast University Press; 2006 246-270 p.

[5] Yong L, Xueyan M. Applied Immunohematology: Blood Group Theory and Experimental Techniques. Beijing: Science Press; 2006.

[6] Geoff D. Human Blood Groups. Second Edition. UK: Blackwell Science Ltd a Blackwell Publishing Company; 2002. 39-42 p.

[7] Yamaguchi H, Okubo Y, Hazama F. Another Japanese A2B3 bloodgroup family with the propositus having O-group father. Proc Jpn
Acad. 1966; 42(1): 517-520. https://doi.org/10.2183/pjab 1945.42 .517

[8] Yuqin S, Xiaoli W, Di S, et al. Analysis on a case of cisAB family. Chinese Journal of Blood Transfusion. 2013; 26(1): 82.

[9] Xiaohua W, Ling M, Enbo W, et al. Serology and genotype analysis of CisAB01 alleles (with 2 cases report). Journal of Clinical Hematology. 2017; 30(2): 161-163.

[10] Yamamoto F, Mcneill PD, Kominato Y, et al. Molecular genetic analysis of the ABO blood group system: 2. Cis-AB alleles. Vox Sang. 1993; 64(2): 120-123. PMid:8456556. https ://doi.org/10.115 $9 / 000462322$

[11] Zhonghui G, Dong X, Ziyan Z, et al. B (A) 641T $>C$ alleles found in Chinese Han population. Chinese Journal of Blood Transfusion. 2006; 19(1): 17-20.

[12] Wenhua Z, Caimei Z. Detection analysis on individual cases of cisAB and AxB blood groups. Journal of Clinical Transfusion and Laboratory Medicine. 2014; 16(4): 434-435.

[13] Beiyuan W, Fan Y, Lixin J. A Case of Discrepancy of ABO blood grouping caused by AXB genotype. Chinese Journal of Laboratory Diagnosis. 2010; 14(12): 2054-2055. 